\title{
Residual effect of two insecticides and neem oil against epilachna beetle, Epilachna vigintioctopunctata (Fab.) on bitter gourd
}

\author{
M. Mala ${ }^{1}$, M. M. U. Islam ${ }^{2}$ and K. S. Islam² \\ ${ }^{1}$ Department of Entomology, Patuakhali University of Science and Technology, ${ }^{2}$ Department of Entomology, \\ Bangladesh Agricultural University, Mymensingh-2202, Bangladesh. E-mail: mukta015@gmail.com
}

\begin{abstract}
Experiments were conducted in the Laboratory and Entomology Field Laboratory to determine the residual effect of two insecticides (viz. Siperin 10EC, Malathion 57EC) and a botanical (Neem oil) against Epilachna beetle, Epilachna vigintioctopunctata (Fab.) during the period from February to May 2009. To evaluate the residual effect of one synthetic and one organophosphors pesticides and one botanical pesticide on the mortality of Epilachna beetle, different concentrations of the insecticides (1.0, 2.0 and 3.0\% of Neem oil, 1.0, 1.2 and 1.5\% of Malathion and 0.5, 1.0 and 1.5\% of Siperin.) was used. The mortality of the beetles was recorded at 4, 5, 6 and 7 days after treatments. After 7 days the highest mortality was found on first instar larvae (70.0 in laboratory condition and 65.0 in field condition) treated by Siperin 10EC (1.5\% conc.) and the lowest mortality was found on adults Epilachna beetle (17.5 in laboratory condition and 15.0 in field condition) treated by Neem oil $(1.0 \%$ conc.) The results indicated that Siperin 10EC has longer residual effect than Malathion 57EC and Neem oil. The effectivity ranked: Siperin > Malathion > Neem oil.
\end{abstract}

Keywords: Residual effect, Epilachna vigintioctopunctata, Siperin, Malathion, Neem oil

\section{Introduction}

Among the important pest species that feed on cucurbits, Epilachna beetle is the most destructive specially in South Asia. It is widely distributed in Asia, Europe, Australia, America, and West Indies. This beetle feeds exclusively on the plants belonging to the Cucurbitacae family and plants of other families. Both the grub and adult feed on the epidermal tissues of leaves by scraping surface and damage up to $80 \%$ of plants (Rajagopal and Trivedi, 1989). Thirty five to seventy five percent leaves were severely damaged by grubs and adults (Srivastava and Katiyar, 1991). The grubs confine their attack to the lower surface of the leaves and adults usually feed on the upper surface of the leaves (Pradhan et al., 1990).

Insecticides are the major means of insect control in all crops including vegetables in Bangladesh. But the increasing trend of using insecticides has given rise to many problems. To solve these problems farmer can shift the practice of sole reliance of insecticide to alternative approaches. The biologically active natural plant products may play a significant role in this regard. There are about 2000 plant species that posses pest control properties (Ahmed, 1984). The indigenous plant materials are available everywhere in our country and can easily be produced by the farmers and small traders. Among them neem oil has extensively been used and has proved its pest controlling efficacy against several insect pests both in field and storage (Saxena et al. 1981; Heyde et al., 1983). Neem oil has no adverse effects in the argoecosystem and its cost of production is likely to be less compared to that of chemical insecticide (Saxena et al. 1981). Neem oil is used as a pesticide, insecticide, fungicide and miticide. They also have antifeedant, repellent and growth inhibitory properties (Kumar and Babu, 1998).

The present study was undertaken to investigate the residual effect of Neem oil, Malathion and Siperin for controlling epilachna beetle under laboratory and field condition.

\section{Materials and Methods}

The experiment was carried out in the laboratory of the Department of Entomology and Entomology Field Labotary, Bangladesh Agricultural University (BAU), Mymensingh, during February to May, 2009. Details of the materials and methods of the study are presented below:

In order to meet the supply of the test insect, a stock culture of large number of grubs and adult beetles of Epilachna vigintioctopunctata was maintained in the laboratory and in the field on bitter gourd plant. 
Neem oil, Malathion57EC and Siperin10EC were collected from Department of Entomology, Bangladesh Agricultural University, Mymensingh. From these insecticides different concentrations were prepared with water. The mortality of $E$. vigintioctopunctata was evaluated by exposing first, second, third instars larvae and adult separately in Petri dish containing bitter gourd leaves treated with 1.0, 2.0 and 3.0\% of Neem oil, $1.0,1.20$ and $1.5 \%$ of Malathion and $0.5,1.0$ and $1.5 \%$ of Siperin. Ten larvae and adult of $E$. vigintioctopunctata were supplied in each petri dish for each concentration. In control treatment, leaves were treated with distilled water only. The leaf petiole was covered with water soaked cotton pad to prevent water loss. And it was also performed in the field and in case of control plot there was no any insecticide application performed.

Residual toxicity effect was evaluated by observing mortality of insects released in the treated plants and petri dishes at 4 days after treatment (DAT). Data were recorded at 4, 5, 6 and 7 days after treatment (DAT). The experiment was repeated for 3 times. The experiments laid out in a Randomized Complete Block Design (RCBD). The significance of difference between pair of means was performed by least significance difference (LSD) test taking the probability level $1 \%$ as the maximum unit of significance. Dose response relationships were determined using Duncan's New Multiple Range Test.

\section{Results and Discussion}

Residual effect of insecticides on first instar larvae of Epilachna beetle, E. vigintioctopunctata (Fab.)

The results revealed the highest mortality on first instar larvae of E. vigintioctopunctata in Siperin10EC treated bitter gourd leaf. The cumulative mortality was significantly higher in siperin10EC than other insecticides. The residual toxicity remains up to 7 days after treatment in case of Siperin. Considering the cumulative percent mortality the highest residual toxicity was recorded at $1.5 \%$ conc. Mortality or residual toxicity was significant higher in laboratory condition (27.5\%) than field condition (22.5\%) (Table 1).

There were significant differences among the treatments. Ranking order of insecticides for residual effect was: Siperin > Malathion > Neem oil

Table 1. Residual effect of insecticides on first instar larvae of Epilachna beetle, $E$. vigintioctopunctata (Fab.) at different time interval

\begin{tabular}{|c|c|c|c|c|c|c|c|c|c|}
\hline \multirow{3}{*}{ Insecticides } & \multirow{3}{*}{$\begin{array}{c}\text { Concentration } \\
(\%)\end{array}$} & \multicolumn{8}{|c|}{ Cumulative mortality ( $\%$ ) of first instar larvae at different time interval } \\
\hline & & \multicolumn{4}{|c|}{ Laboratory condition } & \multicolumn{4}{|c|}{ Field condition } \\
\hline & & 4 days & 5 days & 6 days & 7 days & 4 days & 5 days & 6 days & 7 days \\
\hline \multirow{3}{*}{ Malathion 57EC } & 1.0 & $12.5 \mathrm{bc}$ & $20.0 \mathrm{~d}$ & $32.5 \mathrm{~cd}$ & 40.0 de & $10.0 \mathrm{bcd}$ & $15.0 \mathrm{bc}$ & $25.0 \mathrm{~cd}$ & $32.5 \mathrm{~cd}$ \\
\hline & 1.2 & $20.0 \mathrm{ab}$ & $35.0 \mathrm{ab}$ & $42.0 \mathrm{ab}$ & $55.0 \mathrm{bc}$ & $17.5 \mathrm{ab}$ & $16.0 \mathrm{bc}$ & $37.0 \mathrm{ab}$ & $45.0 \mathrm{~b}$ \\
\hline & 1.5 & $21.0 \mathrm{ab}$ & $36.0 \mathrm{ab}$ & $45.0 \mathrm{ab}$ & $57.5 .0 \mathrm{~b}$ & $18.5 \mathrm{ab}$ & $20.5 \mathrm{~b}$ & $40.0 \mathrm{ab}$ & $50.0 \mathrm{~b}$ \\
\hline \multirow[t]{3}{*}{ Siperin 10EC } & 0.5 & $17.5 \mathrm{ab}$ & $25.0 \mathrm{bcd}$ & $35.0 \mathrm{bc}$ & $45.0 \mathrm{cde}$ & $12.5 \mathrm{bc}$ & $20.0 \mathrm{~b}$ & $30.0 \mathrm{bc}$ & $42.5 \mathrm{bc}$ \\
\hline & 1.0 & $20.00 \mathrm{ab}$ & $32.5 \mathrm{abc}$ & $45.0 \mathrm{ab}$ & $57.5 \mathrm{~b}$ & $15.0 \mathrm{abc}$ & $30.0 \mathrm{a}$ & $40.0 \mathrm{ab}$ & $47.5 \mathrm{~b}$ \\
\hline & 1.5 & $27.5 \mathrm{a}$ & $42.5 \mathrm{a}$ & $55.0 \mathrm{a}$ & $70.0 \mathrm{a}$ & $22.5 \mathrm{a}$ & $35.0 \mathrm{a}$ & $50.00 \mathrm{a}$ & $65.0 \mathrm{a}$ \\
\hline \multirow{3}{*}{ Neem oil } & 1.0 & $2.5 \mathrm{C}$ & $12.5 \mathrm{~d}$ & $22.5 \mathrm{~d}$ & $27.5 \mathrm{f}$ & $2.5 \mathrm{~d}$ & $10.0 \mathrm{c}$ & $17.5 \mathrm{~d}$ & $22.5 \mathrm{~d}$ \\
\hline & 2.0 & $10.0 \mathrm{bc}$ & $22.5 \mathrm{~cd}$ & $30.0 \mathrm{~cd}$ & 37.5 ef & $7.5 \mathrm{~cd}$ & $17.5 \mathrm{bc}$ & $25.0 \mathrm{~cd}$ & $32.5 \mathrm{~cd}$ \\
\hline & 3.0 & $12.5 \mathrm{bc}$ & $20.0 \mathrm{~cd}$ & $35.0 \mathrm{bc}$ & $50.0 \mathrm{bcd}$ & $10.0 \mathrm{bcd}$ & $17.5 \mathrm{bc}$ & $30.0 \mathrm{bc}$ & $42.5 \mathrm{bc}$ \\
\hline Control & & 0.0 & 0.0 & 0.0 & 0.0 & 0.0 & 0.0 & 0.0 & 0.0 \\
\hline
\end{tabular}

\# Data in a column followed by different letter(s) are significantly different at $\mathrm{p}<0.01$ by DMRT. \#10 larvae were used per treatment. \# 3 replications were made. \# Control treatment was not included in analysis

Residual effect of insecticides on second instar larvae of Epilachna beetle, E. vigintioctopunctata (Fab.)

The results revealed the highest mortality on second instar larvae of E. vigintioctopunctata in Siperin10EC treated bitter gourd leaf. The cumulative mortality was significantly higher in siperin10EC than other insecticides. The residual toxicity remains up to 7 days after treatment in case of Siperin. Considering the cumulative percent mortality the highest residual toxicity was recorded at $1.5 \%$ conc. Mortality or residual toxicity was significant higher in laboratory condition (22.5\%) than field condition (20.0\%) (Table 2). 
There were significant differences among the treatments. Ranking order of insecticides for residual effect was: Siperin > Malathion > Neem oil.

Table 2. Residual effect of insecticides on second instar larvae of Epilachna beetle, $E$. vigintioctopunctata (Fab.) at different time interval

\begin{tabular}{|c|c|c|c|c|c|c|c|c|c|}
\hline \multirow{3}{*}{ Insecticides } & \multirow{3}{*}{$\begin{array}{c}\text { Concentration } \\
(\%)\end{array}$} & \multicolumn{8}{|c|}{ Cumulative mortality ( $\%$ ) of second instar larvae at different time interval } \\
\hline & & \multicolumn{4}{|c|}{ Laboratory condition } & \multicolumn{4}{|c|}{ Field condition } \\
\hline & & 4 days & 5 days & 6 days & 7 days & 4 days & 5 days & 6 days & 7 days \\
\hline \multirow{3}{*}{ Malathion 57EC } & 1.0 & $12.5 \mathrm{bcd}$ & $17.5 \mathrm{~d}$ & 22.5 ef & $30.0 \mathrm{~d}$ & $5.0 \mathrm{~b}$ & $12.5 \mathrm{C}$ & $20.0 \mathrm{~cd}$ & $27.5 \mathrm{e}$ \\
\hline & 1.2 & $17.5 \mathrm{ab}$ & $32.5 \mathrm{abc}$ & $42.5 \mathrm{abc}$ & $52.5 \mathrm{~b}$ & $12.0 \mathrm{ab}$ & $25.5 \mathrm{ab}$ & $32.5 \mathrm{~b}$ & $42.5 \mathrm{bc}$ \\
\hline & 1.5 & $18.5 \mathrm{ab}$ & $35.0 \mathrm{ab}$ & $45.0 \mathrm{ab}$ & $60.0 \mathrm{ab}$ & $15.0 \mathrm{ab}$ & $27.5 \mathrm{ab}$ & $40.0 \mathrm{ab}$ & $52.5 \mathrm{ab}$ \\
\hline \multirow[t]{3}{*}{ Siperin 10EC } & 0.5 & $15.0 \mathrm{abc}$ & $22.5 \mathrm{~cd}$ & $32.5 \mathrm{cde}$ & $42.5 \mathrm{C}$ & $12.5 \mathrm{ab}$ & $20.0 \mathrm{bc}$ & $30.0 \mathrm{bc}$ & $37.5 \mathrm{~cd}$ \\
\hline & 1.0 & $17.5 \mathrm{ab}$ & $30.0 \mathrm{abc}$ & $37.5 \mathrm{bc}$ & $55.0 \mathrm{~b}$ & $15.0 \mathrm{ab}$ & $25.0 \mathrm{ab}$ & $35.0 \mathrm{ab}$ & $47.5 \mathrm{abc}$ \\
\hline & 1.5 & $22.5 \mathrm{a}$ & $37.5 \mathrm{a}$ & $52.5 \mathrm{a}$ & $67.0 \mathrm{a}$ & $20.0 \mathrm{a}$ & $30.0 \mathrm{a}$ & $45.0 \mathrm{a}$ & $55.0 \mathrm{a}$ \\
\hline \multirow{3}{*}{ Neem oil } & 1.0 & $5.0 \mathrm{~d}$ & $15.0 \mathrm{~d}$ & $20.0 \mathrm{f}$ & $25.0 \mathrm{~d}$ & $5.0 \mathrm{~b}$ & $12.5 \mathrm{c}$ & $15.0 \mathrm{~d}$ & $22.5 \mathrm{e}$ \\
\hline & 2.0 & $7.5 \mathrm{~cd}$ & $17.5 \mathrm{~d}$ & 25.0 def & $30.0 \mathrm{~d}$ & $5.0 \mathrm{~b}$ & $15.0 \mathrm{c}$ & $20.0 \mathrm{~cd}$ & $27.5 \mathrm{de}$ \\
\hline & 3.0 & $12.5 \mathrm{bcd}$ & $25.0 \mathrm{bcd}$ & $35.0 \mathrm{bcd}$ & $45.0 \mathrm{c}$ & $10.0 \mathrm{ab}$ & $20.0 \mathrm{bc}$ & $30.0 \mathrm{bc}$ & $40.0 \mathrm{c}$ \\
\hline Control & & 0.0 & 0.0 & 0.0 & 0.0 & 0.0 & 0.0 & 0.0 & 0.0 \\
\hline
\end{tabular}

\# Data in a column followed by different letter(s) are significantly different at $\mathrm{p}<0.01$ by DMRT. \#10 larvae were used per treatment. \# 3 replications were made. \# Control treatment was not included in analysis

Residual effect of insecticides on third instar larvae of Epilachna beetle, E. vigintioctopunctata (Fab.)

The results revealed the highest mortality on third instar larvae of E. vigintioctopunctata in Siperin10EC treated bitter gourd leaf. The cumulative mortality was significantly higher in siperin10EC than other insecticides. The residual toxicity remains up to 7 days after treatment in case of Siperin. Considering the cumulative percent mortality the highest residual toxicity was recorded at $1.5 \%$ conc. Mortality or residual toxicity was significant higher in laboratory condition (20.0\%) than field condition (17.5\%) (Table 3).

There were significant differences among the treatments. Ranking order of insecticides for residual effect was: Siperin > Malathion > Neem oil.

Table 3. Residual effect of insecticides on third instar larvae of Epilachna beetle, $E$. vigintioctopunctata (Fab.) at different time interval

\begin{tabular}{|c|c|c|c|c|c|c|c|c|c|}
\hline \multirow{3}{*}{ Insecticides } & \multirow{3}{*}{$\begin{array}{c}\text { Concentration } \\
(\%)\end{array}$} & \multicolumn{8}{|c|}{ Cumulative mortality ( $\%$ ) of third instar larvae at different time interval } \\
\hline & & \multicolumn{4}{|c|}{ Laboratory condition } & \multicolumn{4}{|c|}{$\begin{array}{ll}\text { Field condition } \\
\end{array}$} \\
\hline & & 4 days & 5 days & 6 days & 7 days & 4 days & 5 days & 6 days & 7 days \\
\hline \multirow{3}{*}{ Malathion 57EC } & 1.0 & $10.0 \mathrm{bc}$ & $15.0 \mathrm{~cd}$ & $20.0 \mathrm{de}$ & $25.0 \mathrm{~d}$ & $5.0 \mathrm{~b}$ & $10.0 \mathrm{c}$ & $17.5 \mathrm{~d}$ & $25.0 \mathrm{~d}$ \\
\hline & 1.2 & $12.50 \mathrm{abc}$ & $22.5 \mathrm{bc}$ & $32.5 \mathrm{bc}$ & $55.0 \mathrm{a}$ & $7.5 \mathrm{~b}$ & $17.5 \mathrm{bc}$ & $27.5 \mathrm{bcd}$ & $40.0 \mathrm{bc}$ \\
\hline & 1.5 & $15.0 \mathrm{ab}$ & $25.0 \mathrm{~b}$ & $35.0 \mathrm{~b}$ & $57.5 \mathrm{a}$ & $12.5 \mathrm{ab}$ & $22.5 \mathrm{ab}$ & $32.5 \mathrm{ab}$ & $45.0 \mathrm{~b}$ \\
\hline \multirow[t]{3}{*}{ Siperin 10EC } & 0.5 & $12.5 \mathrm{abc}$ & $20.0 \mathrm{bcd}$ & $30.0 \mathrm{bcd}$ & $40.0 \mathrm{c}$ & $10.0 \mathrm{ab}$ & $17.5 \mathrm{bc}$ & $25.0 \mathrm{bcd}$ & $35.0 \mathrm{c}$ \\
\hline & 1.0 & $15.0 \mathrm{ab}$ & $27.5 \mathrm{ab}$ & $35.0 \mathrm{~b}$ & $52.5 \mathrm{ab}$ & $12.5 \mathrm{ab}$ & $22.5 \mathrm{ab}$ & $30.0 \mathrm{bc}$ & $42.5 \mathrm{bc}$ \\
\hline & 1.5 & $20.0 \mathrm{a}$ & $35.0 \mathrm{a}$ & $50.0 \mathrm{a}$ & $62.5 \mathrm{a}$ & $17.5 \mathrm{a}$ & $30.0 \mathrm{a}$ & $42.5 \mathrm{a}$ & $55.0 \mathrm{a}$ \\
\hline \multirow{3}{*}{ Neem oil } & 1.0 & $5.0 \mathrm{c}$ & $12.5 \mathrm{~d}$ & $17.5 \mathrm{e}$ & $22.5 \mathrm{~d}$ & $5.0 \mathrm{~b}$ & $10.0 \mathrm{c}$ & $17.5 \mathrm{~d}$ & $22.5 \mathrm{~d}$ \\
\hline & 2.0 & $5.0 \mathrm{c}$ & $15.0 \mathrm{~cd}$ & 22.5 cde & $27.5 \mathrm{~d}$ & $5.0 \mathrm{~b}$ & $12.5 \mathrm{bc}$ & $20.0 \mathrm{~cd}$ & $25.0 \mathrm{~d}$ \\
\hline & 3.0 & $10.0 \mathrm{bc}$ & $22.0 \mathrm{bc}$ & $30.0 \mathrm{bcd}$ & $42.5 \mathrm{bc}$ & $7.5 \mathrm{~b}$ & $17.5 \mathrm{bc}$ & $22.5 \mathrm{bcd}$ & $37.5 \mathrm{bc}$ \\
\hline Control & & 0.0 & 0.0 & 0.0 & 0.0 & 0.0 & 0.0 & 0.0 & 0.0 \\
\hline
\end{tabular}

\# Data in a column followed by different letter(s) are significantly different at $\mathrm{p}<0.01$ by DMRT. \#10 larvae were used per treatment. \# 3 replications were made. \# Control treatment was not included in analysis

\section{Residual effect of insecticides on adult Epilachna beetle, E. vigintioctopunctata (Fab.)}

The results revealed the highest mortality on adult Epilachna beetle, E. vigintioctopunctata in Siperin10EC treated bitter gourd leaf. The cumulative mortality was significantly higher in siperin10EC than other insecticides. The residual toxicity remains up to 7 days after treatment in case of Siperin. considering the cumulative percent mortality the highest residual toxicity was recorded at $1.5 \%$ conc. Mortality or residual toxicity was significant higher in laboratory condition (17.5\%) than field condition (15.0\%) (Table 4). 
There were significant differences among the treatments. Ranking order of insecticides for residual effect was: Siperin > Malathion > Neem oil.

Table 4. Residual effect of insecticides on adult epilachna beetle, E. vigintioctopunctata (Fab.) at different time interval

\begin{tabular}{|c|c|c|c|c|c|c|c|c|c|}
\hline \multirow{3}{*}{ Insecticides } & \multirow{3}{*}{ Concentration (\%) } & \multicolumn{8}{|c|}{ Cumulative mortality ( $\%$ ) of adult epilachna beetle at different time interval } \\
\hline & & \multicolumn{4}{|c|}{ Laboratory condition } & \multicolumn{4}{|c|}{$\begin{array}{c}\text { Field condition } \\
\end{array}$} \\
\hline & & 4 days & 5 days & 6 days & 7 days & 4 days & 5 days & 6 days & 7 days \\
\hline \multirow{3}{*}{ Malathion 57EC } & 1.0 & $5.0 \mathrm{bc}$ & $12.5 \mathrm{c}$ & $17.5 \mathrm{~d}$ & $20.0 \mathrm{~d}$ & $5.0 \mathrm{~b}$ & $7.5 \mathrm{~cd}$ & $15.0 \mathrm{de}$ & $20.0 \mathrm{~d}$ \\
\hline & 1.2 & $7.5 \mathrm{bc}$ & $17.5 \mathrm{bc}$ & $25.0 \mathrm{bcd}$ & $35.0 \mathrm{c}$ & $5.0 \mathrm{~b}$ & $15.0 \mathrm{bcd}$ & 17.5 cde & $27.5 \mathrm{c}$ \\
\hline & 1.5 & $12.5 \mathrm{ab}$ & $22.5 \mathrm{~b}$ & $30.0 \mathrm{~b}$ & $42.5 \mathrm{bc}$ & $7.5 \mathrm{ab}$ & $17.5 \mathrm{bc}$ & $25.0 \mathrm{bc}$ & $35.0 \mathrm{~b}$ \\
\hline \multirow[t]{3}{*}{ Siperin 10EC } & 0.5 & $10.0 \mathrm{abc}$ & $17.5 \mathrm{bc}$ & $27.5 \mathrm{bc}$ & $37.5 \mathrm{c}$ & $7.0 \mathrm{ab}$ & $15.0 \mathrm{bcd}$ & $22.5 \mathrm{bcd}$ & $32.5 \mathrm{bc}$ \\
\hline & 1.0 & $12.5 \mathrm{ab}$ & $22.5 \mathrm{~b}$ & $32.5 \mathrm{~b}$ & $47.5 \mathrm{~b}$ & $10.0 \mathrm{ab}$ & $20.0 \mathrm{ab}$ & $27.5 \mathrm{~b}$ & $37.5 \mathrm{~b}$ \\
\hline & 1.5 & $17.5 \mathrm{a}$ & $32.5 \mathrm{a}$ & $47.5 \mathrm{a}$ & $60.0 \mathrm{a}$ & $15.0 \mathrm{a}$ & $27.5 \mathrm{a}$ & $37.5 \mathrm{a}$ & $47.5 \mathrm{a}$ \\
\hline \multirow{3}{*}{ Neem oil } & 1.0 & $2.5 \mathrm{c}$ & $10.0 \mathrm{c}$ & $15.0 \mathrm{~d}$ & $17.5 \mathrm{~d}$ & $2.5 \mathrm{~b}$ & $5.0 \mathrm{~d}$ & $10.0 \mathrm{e}$ & $15.0 \mathrm{~d}$ \\
\hline & 2.0 & $5.0 \mathrm{bc}$ & $12.5 \mathrm{c}$ & $17.5 \mathrm{~cd}$ & $22.5 \mathrm{~d}$ & $5.0 \mathrm{~b}$ & $10.0 \mathrm{bcd}$ & 17.5 cde & $20.0 \mathrm{~d}$ \\
\hline & 3.0 & $10.0 \mathrm{abc}$ & $17.5 \mathrm{bc}$ & $25.0 \mathrm{~b} \mathrm{~cd}$ & $35.0 \mathrm{c}$ & $7.5 \mathrm{ab}$ & $15.0 \mathrm{bcd}$ & $22.5 \mathrm{bcd}$ & $32.5 \mathrm{bc}$ \\
\hline Control & & 0.0 & 0.0 & 0.0 & 0.0 & 0.0 & 0.0 & 0.0 & 0.0 \\
\hline
\end{tabular}

\# Data in a column followed by different letter(s) are significantly different at $\mathrm{p}<0.01$ by DMRT. \#10 larvae were used per treatment. \# 3 replications were made. \# Control treatment was not included in analysis

\section{Comparative Performance of insecticides on epilachna beetle, E. vigintioctopunctata (Fab.)}

After 7days the cumulative mortality (\%) of first, second, third instar larvae and adult Epilachna beetle was highest in Siperin10EC at $1.5 \%$ conc. And the lowest Cumulative mortality (\%) was found in Neem oil at $1.0 \%$ conc. both in laboratory and field condition (Fig.1 and Fig.2)

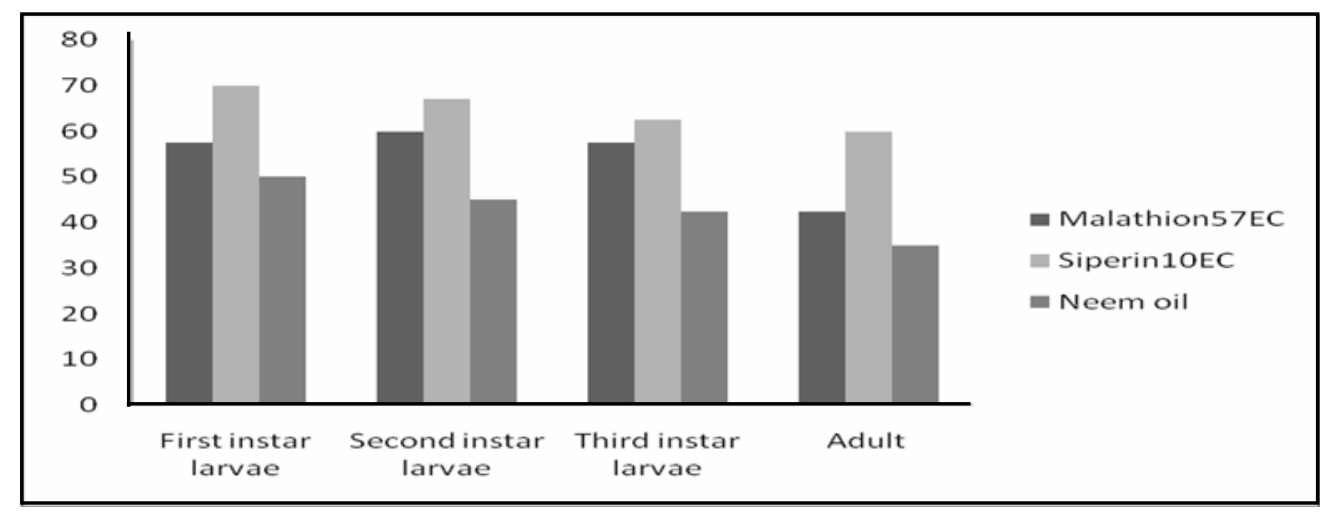

Fig. 1. Comparative performance of insecticides on Epilachna beetle in laboratory condition

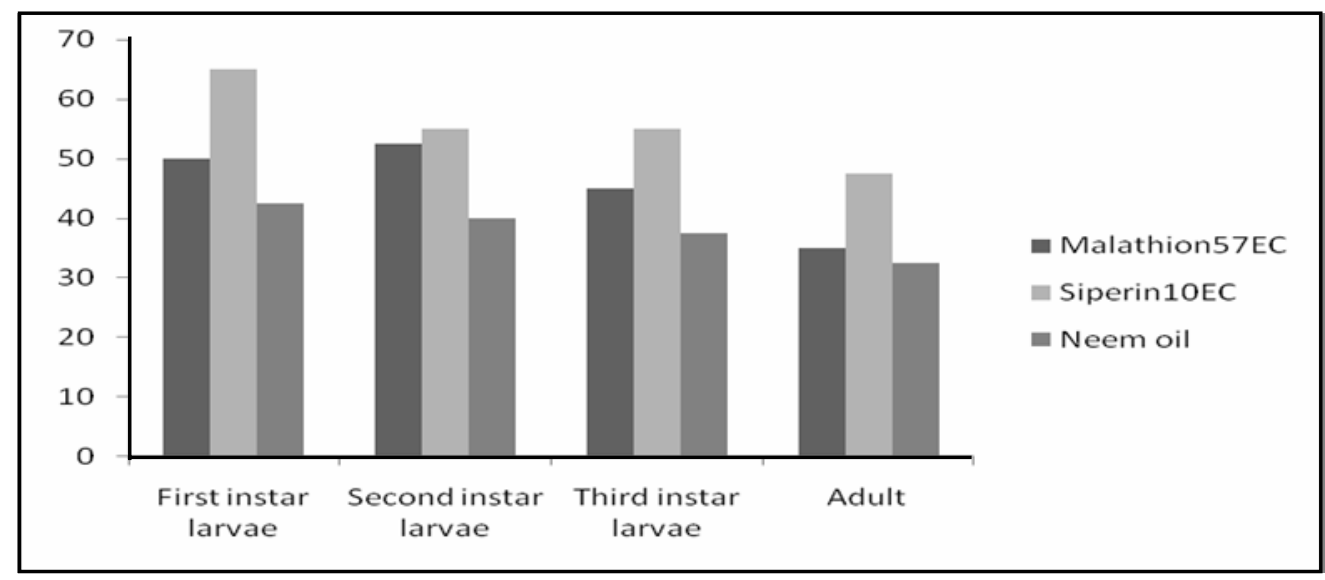

Fig. 2. Comparative performance of insecticides on Epilachna beetle in field condition 
Tewari and Moorthy (1989) carried out a field trails in Karnataka, India, in June 1987 to evaluate the effectiveness of 9 insecticides against Epilachna vigintioctopunctata on brinjal. Malathion at $400 \mathrm{~g}$ a.i. /ha was moderately effective against the pest followed by carbaryl at $1500 \mathrm{~g}$ a.i. /ha. The synthetic pyrethroids deltamethrin, cypermethrin, fenvalerate and permethrin were highly effective against the $E$. vigintioctopunctata from laboratory and field experiments with three insecticides, Siperin, Malathion and Neem oil exhibited different toxic effects on the different stages of the life cycle of Epilachna beetle, Epilachna vigintioctopunctata (Fab).

Karmakar and Bhole (2001) observed the efficacy and persistent toxicity of some neem products such as Neem oil, neembicidine and neemark against adult of Epilachna dodecastigma. The treatments with 2\% neem oil, $2 \%$ neembicidine and $2 \%$ neemark resulted $100 \%, 90.69 \%$ and $71.90 \%$ mortality, respectively. Shanmugapriyan and Kingsly (2001) reported the effects of neem oil at $0.25,0.5$ and $1.5 \%$ on larvae of $E$. vigintioctopunctata. Neem oil at $1.5 \%$ concentration caused the highest mortality of third $(95.23 \%)$ and fourth instars (76.19\%) larvae. Neem oil at 0.25 and $0.5 \%$ concentrations resulted in 57.1 and $85.7 \%$ of second instar, 47.6 and $85.7 \%$ of third instar and 57.1 and $80.9 \%$ mortality of fourth instar larvae.

The present finding indicates that all the three insecticide are effective against larval instar and adult epilachna beetle. These three insecticides caused different residual effect after 4, 5, 6 and 7 days. The rank order were Siperin $>$ Malathion $>$ Neem oil. It showed that Neem oil has less residual toxicity than other two insecticides. Though Neem oil had low mortality rate, its use could be considered safe for man, animals, fish and non target fauna if good practices are followed.

\section{References}

Ahmed, S. 1984. Studies on neem oil: A potential antifeedant and growth inhibitor of epilachna beetle, Epilachna dodecastigma (Wied.) (Coleoptera: Coccinellidae). M.S. Thesis, Dept. of Entomology, Bangladesh Agricultural University, Mymensingh. $68 \mathrm{p}$.

Heyde, J. Vd., Saxena, R.C. and Schmutterer, H. 1983. Neem oil and neem' extracts as potential insecticides for control of Hemipterous rice pests Abs. $2^{\text {nd }}$. Int. Neem cont. Reuisch Holzhausen Castle, FRG, May. p. 27.

Karmakar, M.S. and Bhole, S.R. 2001. Efficacy and persistent toxicity of some neem products against adults of Epilachna dodecastigma (Wied.) Plant Prot. Bull. Faridabad. 53(1-2): 22-25.

Kumar, S.P. and Babu, P.C.S. 1998. Toxicity of neem azal formulations to pupae and adults of Epilachna vigintioctopunctata Fab. (Coleoptera: Coccinellidae). Insect Environ. 3(4): 93-98.

Pradhan, S., Jotwani, M.G. and Prakash, S. 1990. Comparative toxicity of insecticides to the grubs and adults of Epilachna vigintioctopunctata (Fab.) (Coleoptera: Coccinellidae). Indian J. Entomol. 24(4): 223-230.

Rajagopal, D. and Trivedi, T.P. 1989. Status, bioecology and management of epilachna beetle, Epilachna vigintioctopunctata (Fab.) (Coleoptera: Coccinellidae) on potato in India. Trop. Pest. Manag. 35(4): 410-413.

Saxena, R.C., Liqquido, N.J. and Justo, H.D. 1981. Neem seed oil, an antifeedant for brown planthopper. Proc Ist. Int. Neem conf. Rottach. Egern. FRG, June 1980. pp. 171-188.

Shanmugapriyan, R. and Kingsly, S. 2001. Bioefficacy of neem oil on larvae of bitter gourd beetle, Epilachna vigintioctopunctata (Coccinellidae: Coleoptera). Indian J. Ecotoxicol. 11(3-4): 215-219.

Srivastava, A.S. and Katiyar, S.S.L. 1991. Epilachna vigintioctopunctata (Fab.) and E. dodecastigma (Muls.) (Coleoptera: Coccinellidae) as a pest of cow pea. Zeits-fur Angewandte Entomol. 71(2): 169-172.

Tewari, G.C. and Moorthy, P.N.K. 1989. Effectiveness of newer insecticides against epilchna beetle, Epilachna vigintioctopunctata (Fabricius) (Coleoptera: Coccinellidae). Indian J. Agril. Sci. 53(12): 1068-1070. 